\title{
Multilevel Technique to Improve PSNR and MSE in Audio Steganography
}

\author{
Ramandeep Kaur \\ M.Tech (ECE).Indo \\ Global college of \\ Engineering, Abhipur, \\ Mohali-Punjab, India
}

\author{
Jagriti Bhatia \\ A.P (ECE). Indo \\ Global college of \\ Engineering, Abhipur, \\ Mohali-Punjab, India
}

\author{
Hardeep Singh \\ Saini, Ph.D \\ Prof. (ECE).Indo \\ Global college of \\ Engineering, Abhipur, \\ Mohali-Punjab, India
}

\author{
Rajesh Kumar \\ Prof. (ECE).Indo \\ Global college of \\ Engineering, Abhipur, \\ Mohali-Punjab, India
}

\begin{abstract}
Now a day's use of an internet in public masses is growing. So large amount of data is stored in computers and transmitted over networks. Data security is main issue now days. Steganography is one of data hiding technique. In this paper we use a multilevel technique in audio steganography. By using multilevel technique we use three methods instead of one. Multilevel technique has advantage that its decoding is difficult and we can send three messages using single cover object. Here we calculate the peak signal to noise ratio (PSNR) and mean square error (MSE) by using multilevel technique.
\end{abstract}

\section{Keywords}

Steganography, audio steganography, multilevel technique

\section{INTRODUCTION}

Steganography is an information hiding technique in which we hide the existence of secret message. Here we embed the secret data into cover file. Embedding is a technique to fix firmly in surrounding mass, e.g. to embed nail into wood [12].

\section{Audio steganography:}

If we embed secret data into digital audio file that technique is called audio steganography. Here we change binary Sequence of digital audio file for embedding secret message. Audio file can be WAV, AU, or MP3 sound files [11]. Audio steganography is more difficult than other methods of steganography. Audio steganography is more challenging than others because audio files are larger than images or text and characteristics of human auditory system (HAS) like large power, dynamic range of hearing and large range of audible frequency[10]. HAS can perceive sound over a range of power greater than $10^{9}$ to 1 and range of frequency greater than $10^{3}$ to $1[10]$.

\section{Multilevel technique:}

Multilevel technique is based on combining two or more steganographic methods such a way that one method is a carrier for other method [2]. In multilevel technique layered approach is used. It is beneficial because of its difficult decoding or we can hide more than one message in a single audio file.

\section{Least significant bit coding (LSB):}

This is the simple and fast method of audio steganography [1]. Here we replace the least significant bits of digital audio files with the message bits. It is very easy to combine with other techniques and provide very high channel capacity [2]. But it has disadvantage that it has low robustness against attacks.

\section{Parity bit coding:}

In parity bit coding method secret message is hiding into the sample regions parity bit [1]. Firstly breaks down the signal into separate regions of samples and encode each bit from secret message in a sample regions parity bit. If the parity bit of sample region does not match with secret message bit then process flips the least significant bit of the samples in the region [1]. It provides more choice to the sender for encoding the secret message [2]. It has disadvantage that it is also not robust.

\section{Spread spectrum method (SS):}

Spread spectrum method spreads the secret message bits across the digital audio signals frequency spectrum. It uses a code that is independent of actual signal [1]. Spread spectrum is of two versions.

- $\quad$ Direct sequence(DSSS)

- Frequency hopping(FSSS)

\section{PROPOSED METHOD}

Here three secret messages can be sent using a single audio file as a cover. Stego file of one level works as a carrier for other next level. Here we combine least significant bit coding (LSB), Parity bit coding, spread spectrum coding (SS). In spread spectrum (SS) we use frequency hopping spread spectrum (FHSS).

Here at the first level secret message (S1) is embedded in carrier file (C1) using least significant bit coding (LSB). Firstly we read the audio file and convert it into digital form and then read the secret message and convert it into digital form and the hide the message bits into least significant bits of audio file. The decoy file (C2) is produced after first level, which act as a cover file for second level. Then we embed secret message (S2) in decoy file (C2) using parity bit coding. Here we read the secret message (S2) and hide the bits of secret message into parity bit of decoy file (C2). The decoy file (C3) is produced after second level. Now it acts as a cover file for level three. Now we embed message (S3) in decoy file (C3) using frequency hopping spread spectrum coding (FHSS). Here we read the secret message (S3) and spread the message bits into decoy file (C3). Three levels of steganography can be identified as layer 1, layer 2 and layer 3 . At layer 1 LSB modification technique and at layer 2 parity encoding technique and at layer 3 Spread Spectrum technique has been used. 


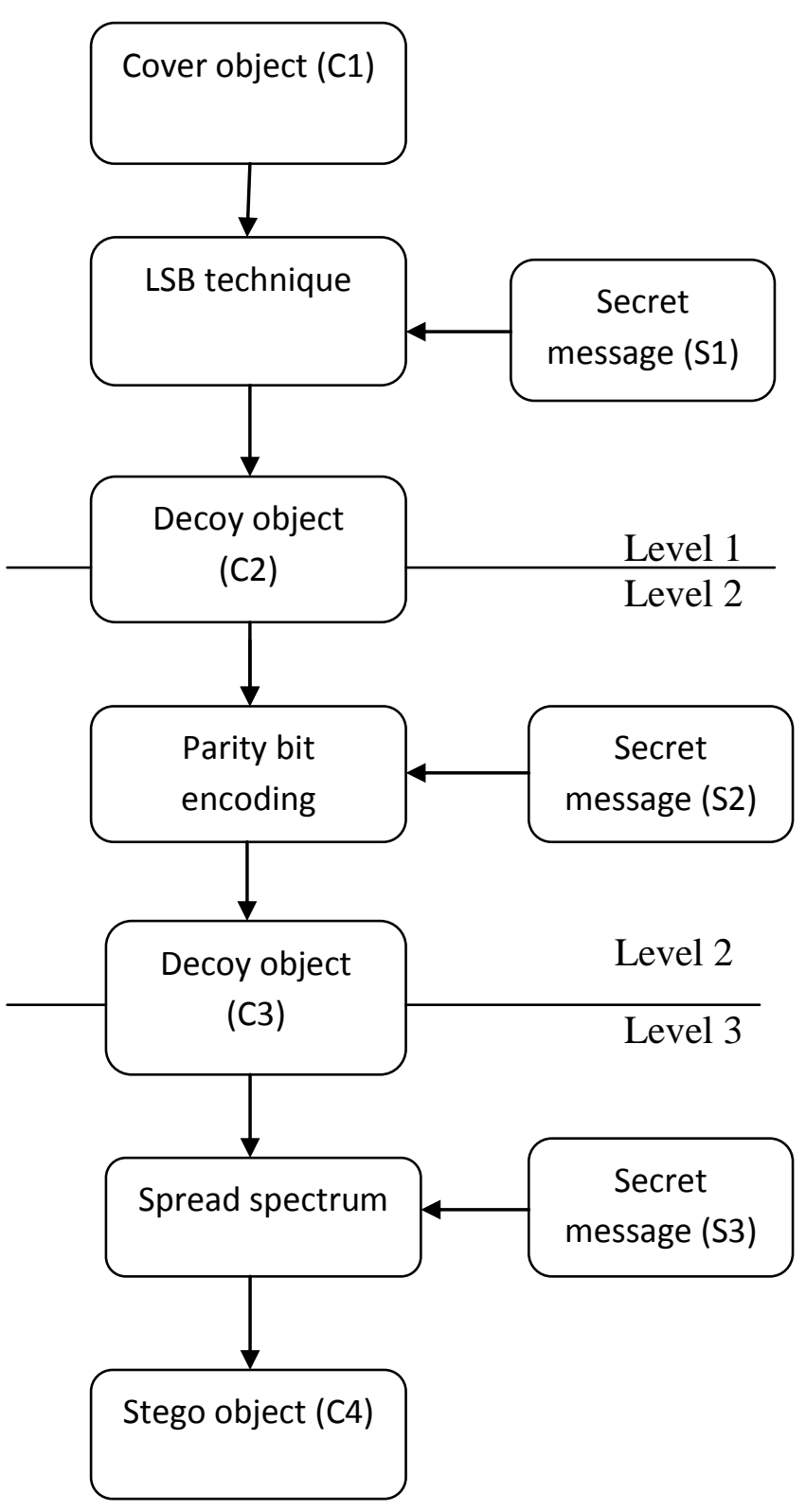

Fig 1: flow chart of Multi-Level Technique

\section{RESULTS}

This paper proposes the audio steganography using multilevel technique. By using multilevel technique we combine least significant bit (LSB) coding, Parity bit coding, spread spectrum coding (SS). In Fig. 2, 3, 4, we can test the performance of by comparing both original audio file with the embedded audio file at all first second or third level respectively. The figure $2,3,4$ shows that there is a no difference between original audio file at all three levels and embedded audio file.

In this, results of all the intermediate steps of the proposed methods are highlighted. Implementation is done on MATLAB Experimental results show the efficiency of the proposed approach.

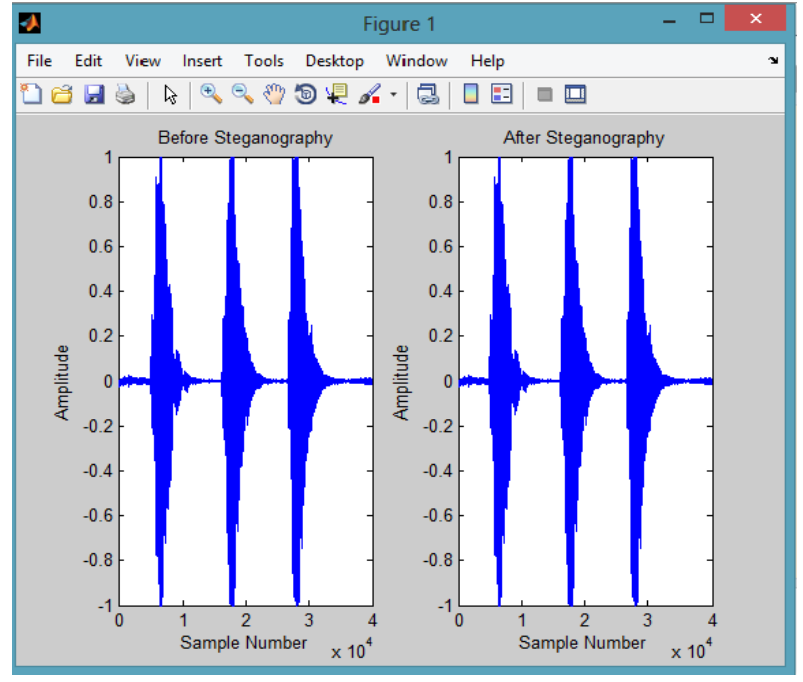

Fig 2: graph of audio file before and after steganography at level 1

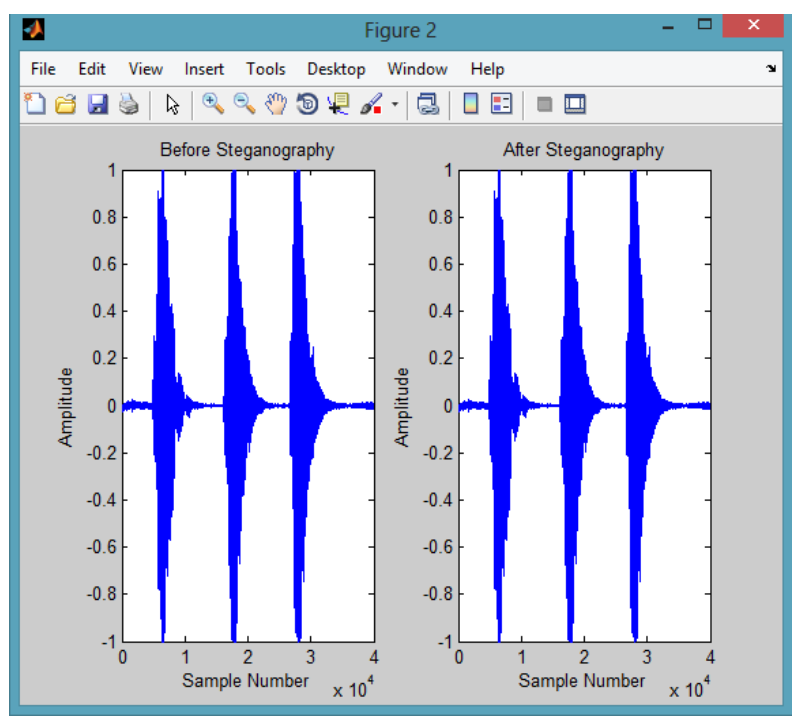

Fig 3: Graph of audio file before and after steganography

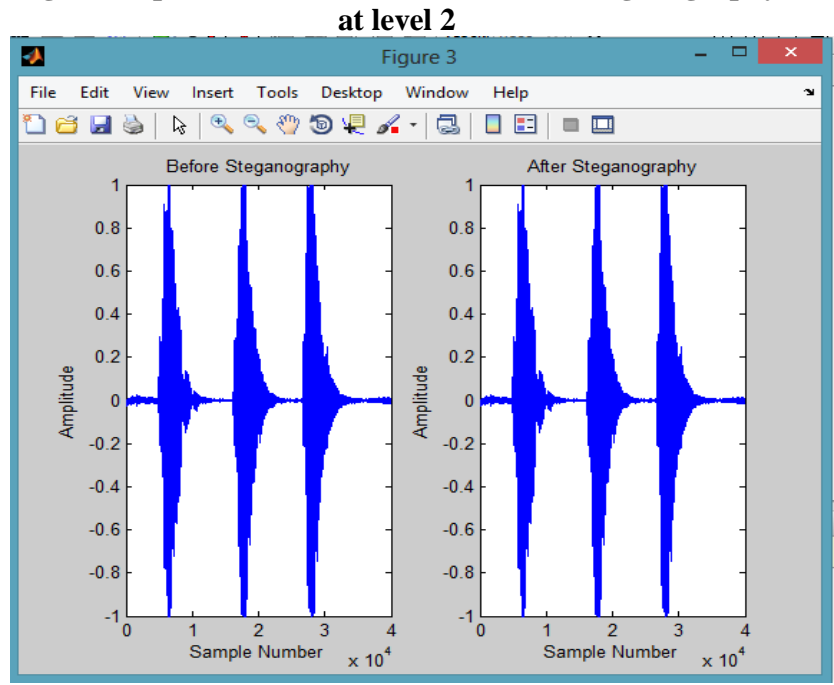

Fig 4: graph of audio file before and after steganography at level 3 
So figure 4 shows that there is no distortion even after embedding third message in third level. The stego audio can be compared by using PSNR and MSE graphs.

\section{Peak Signal to Noise Ratio (PSNR):}

It measures the quality of audio signal. PSNR compare the original audio signal with stego signal [1]. PSNR is measured in decibels $(\mathrm{db})$.

PSNR $=10 \log _{10} \frac{\sum_{n=0}^{N} x(n)^{2}}{\sum_{n=0}^{N}[x(n)-y(n)]^{2}}$

Here $\mathrm{x}(\mathrm{n})$ represent cover audio file and $\mathrm{y}(\mathrm{n})$ is stego audio signal.

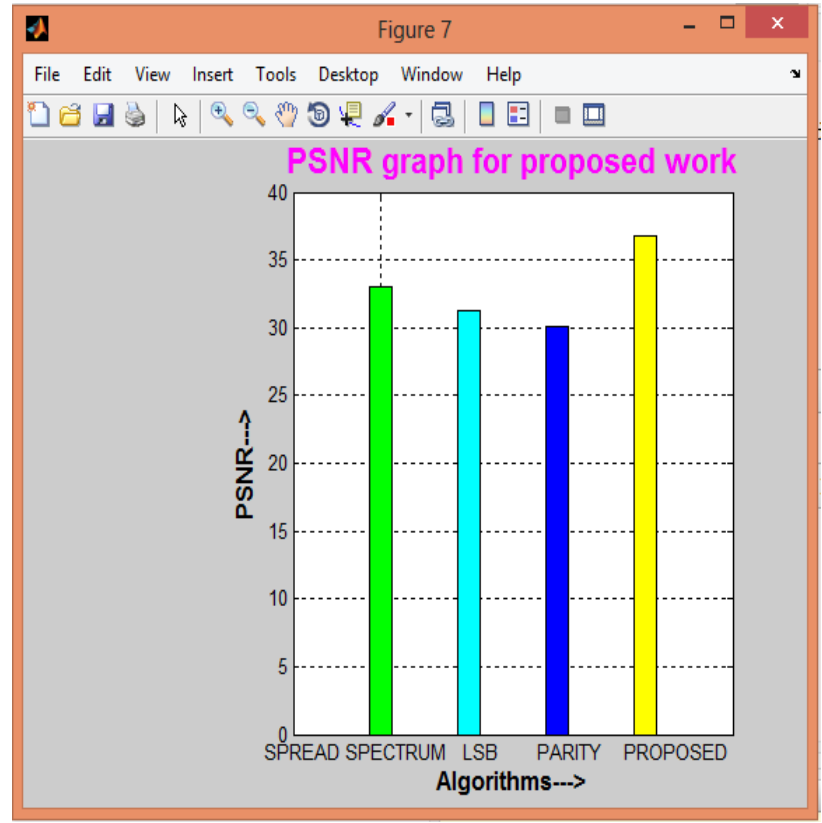

Fig 5: improved value of PSNR

Here figure 5 shows that peak signal to noise ratio (PSNR) of least significant bit coding (LSB), Parity bit coding, spread spectrum coding (SS) is poor than the proposed technique (proposed technique is multilevel technique in which we combine the LSB, parity, SS coding methods).

\section{Mean square error (MSE):}

It measures the distortion in the audio signal. It defines the square of error between original audio signal and stego audio signal [1].

$$
\text { MSE }=10 \log _{10} \sum_{\mathrm{n}=0}^{\mathrm{N}}[\mathrm{x}(\mathrm{n})-\mathrm{y}(\mathrm{n})]^{2}
$$

Here $x(n)$ represents cover audio file and $y(n)$ represents stego audio file.

Here figure 6 shows that mean square error of least significant bit (LSB), parity bit coding, spread spectrum coding (SS), is higher than the proposed technique (multilevel technique).

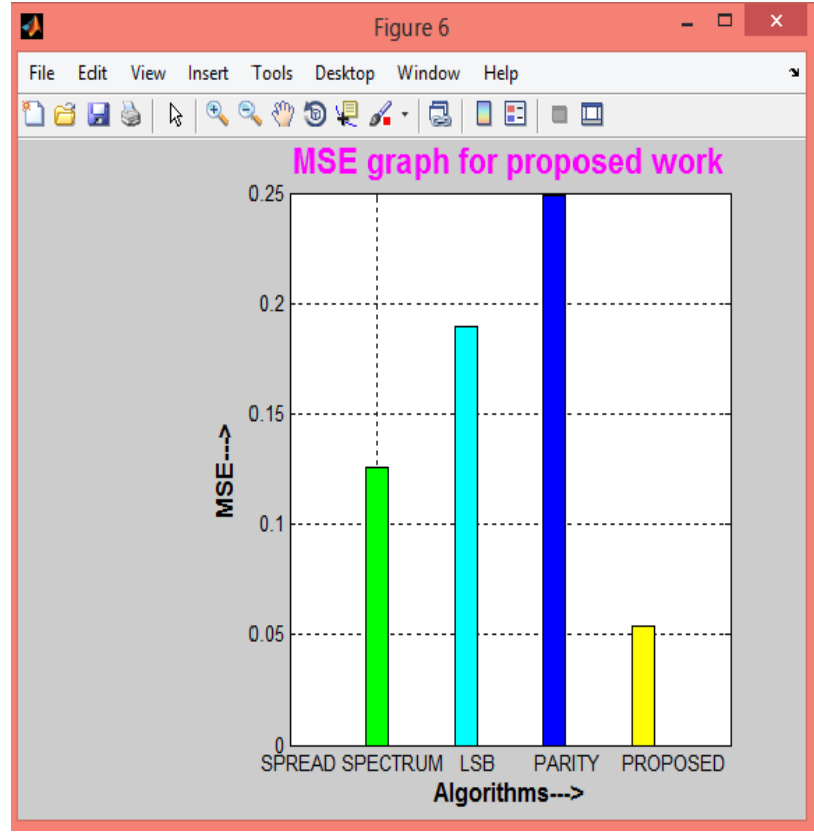

Fig 6: Improved value of MSE

\section{CONCLUSION AND FUTURE SCOPE}

In this paper three traditional methods are combined using multilevel technique. By using multilevel technique we can send three messages through single audio file or it also makes difficult decoding of output audio file. This paper also shows that peak signal to noise ratio (PSNR), mean square error (MSE) of combined technique is better rather than that when we use the least significant bit coding (LSB), parity bit coding, spread spectrum coding (SS)

Further we can extend this approach by combining any four techniques or more. By combining more techniques we can increase capacity and the system becomes more secure towards detection.

\section{REFERENCES}

[1] Pooja P. Balgurgi, Prof. Sonal K. Jagtap, "Intelligent Processing: An Approach of Audio Steganography", 2012 International Conference on Communication, Information \& Computing Technology (ICCICT), Oct. 19-20, Mumbai, India

[2] Kamalpreet kaur, Deepankar Verma, " Multi-Level Steganographic Algorithm for Audio Steganography using LSB, Parity Coding and Phase Coding Technique", International Journal of Advanced Research in Computer Science and Software Engineering Volume 4, Issue 1, January 2014

[3] Kaliappan Gopalan, Qidong Shi “Audio Steganography using Bit Modification - A Tradeoff on Perceptibility and Data Robustness for Large Payload Audio Embedding", IEEE 2010

[4] Masahiro Wakiyama, Yasunobu Hidaka, Koichi Nozaki, "A Novel Phase Coding Technique for Steganography in Auditive Media", IEEE 2011, Sixth International Conference on Availability, Reliability and Security

[5] Harish Kumar, Anuradha, "Enhanced LSB technique for Audio Steganography", ICCCNT'12 26th_28th July 2012, Coimbatore, India 
[6] Fahimeh Rezaei, Tao Ma, Michael Hempel, Dongming Peng, Hamid Sharif, "An Anti-Steganographic Approach for Removing Secret Information in Digital Audio Data hidden by Spread Spectrum Methods", IEEE ICC 2013 Communication and Information Systems Security Symposium

[7] Saswati Ghosh, Debashis De and Debdatta Kandar," A Double Layered Additive Space Sequenced Audio Steganography Technique for Mobile Network", IEEE 2012 International Conference on Radar, Communication and Computing (ICRCC), SKP Engineering College, Tiruvannamalai, TN., India. 21 - 22 December, 2012. Pp.29-33.

[8] Ashwini Mane, Gajanan Galshetwar and Amutha Jeyakumar, "DATA HIDING TECHNIQUE: AUDIO STEGANOGRAPHYUSING LSB TECHNIQUE", International Journal of Engineering Research and Applications Vol. 2, Issue 3, May-Jun 2012 (IJERA).

[9] Ankit Chadha, Neha Satam and Rakshak Sood, Dattatray Bade, " An Efficient Method for Image and Audio
Steganography using Least Significant Bit (LSB) Substitution", International Journal of Computer Applications (0975 - 8887) Volume 77- No.13, September 2013.

[10] Prof. Samir Kumar, BandyopadhyayBarnali, Gupta Banik. "LSB Modification and Phase Encoding Technique of Audio Steganography Revisited", International Journal of Advanced Research in Computer and Communication Engineering Vol. 1, Issue 4, June 2012

[11] Sheelu," Enhancement of Data Hiding Capacity in Audio Steganography", IOSR Journal of Computer Engineering (IOSR-JCE) e-ISSN: 2278-0661, p- ISSN: 22788727Volume 13, Issue 3 (Jul. - Aug. 2013), PP 30-35.

[12] Ramandeep kaur, Jitender Sharma," INFORMATION HIDING BY USING MULTIPLE TECHNIQUES OF AUDIO STEGANOGRAPHY", Vol. 1, Spl. Issue 2 (May, 2014), e-ISN: 1694-2310 | p-ISN: 1694-2426 GV/ICRTEDC/30 